\title{
Mise au point d'une méthode de laboratoire de prévision de la dégradabilité des protéines alimentaires des aliments concentrés dans le rumen
}

\author{
Jocelyne AUFRERE, Dominique CARTAILLER \\ INRA, Station de Recherches sur la Nutrition des Herbivores \\ Centre de Recherches de Clermont-Ferrand-Theix - Saint-Genès-Champanelle, F 63122 Ceyrat
}

\begin{abstract}
Résumé
La quantité de protéines d'origine alimentaire arrivant dans l'intestin grêle du ruminant dépend de la teneur en matières azotées (M.A.T.) des aliments et de la dégradabilité des matières azotées dans le rumen. Celle-ci est estimée à partir de la cinétique de dégradation in sacco dans le rumen (DT) en admettant un taux de sortie des particules de $0,06 \mathrm{~h}^{-1}$. Des méthodes de laboratoire plus faciles à mettre en đuvre et moins coûteuses peuvent être utilisées pour apprécier la dégradabilité dans le rumen de l'azote des aliments.

La méthode proposée consiste en une protéolyse aux temps $1 \mathrm{~h}$ et $24 \mathrm{~h}$ par une protéase d'origine bactérienne dans un tampon borate-phosphate à $\mathrm{pH} 8$. La concentration retenue en enzyme (Sigma type XIV) a été de $1 \mathrm{mg} / 50 \mathrm{ml}$ de tampon pour une quantité fixe de substrat plutôt qu'avec un substrat iso-azoté. L'influence du $\mathrm{pH}$, de la nature de la solution tampon, d'un pré-traitement a également été testée. La prévision de la DT avec cette enzyme est plus précise qu'avec les autres enzymes testées : "protéase Merck " et «ficine ».

Appliquée à 12 aliments, cette méthode de laboratoire permet de prévoir la DT avec une précision meilleure que la solubilité dans un tampon phosphate à $\mathrm{pH} 6,9$ (habituellement utilisée) ( $\mathrm{Sr}=7,0$ contre 12,3 avec la méthode de solubilité).
\end{abstract}

Mots clés: Aliments concentrés, ruminant, dégradabilité théorique, matières azotées, méthode enzymatique.

\section{Introduction}

Le système PDI, comme la plupart des systèmes d'évaluation récents de la valeur azotée des aliments, nécessite de connaître la proportion d'azote alimentaire dégradé dans le rumen. La détermination précise de la dégradation in vivo des protéines dans le rumen est délicate à quantifier, en raison de la difficulté à distinguer dans l'azote arrivant au duodénum l'azote alimentaire de celui des microbes et de l'azote endogène. Des méthodes plus simples d'estimation de la dégradation des protéines ont été développées ; on peut les classer en trois catégories :

- les méthodes chimiques faisant intervenir la solubilité de l'azote, 
- les méthodes utilisant les micro-organismes du rumen (méthodes de dégradabilité « in sacco» et « in vitro »),

- les méthodes enzymatiques.

La plupart des techniques utilisées pour estimer la dégradation des protéines dans le rumen sont basées sur la solubilité dans des solvants qui varient par leur nature, leur $\mathrm{pH}$, la température du milieu, la durée d'extraction (Henderickx et Martin, 1963 ; Wohlt, Sniffen et Hoover, 1973 ; Crooker et al., 1978 ; Waldo et Goering, 1979 ; Krishnamoorthy et al., 1982). Ces méthodes, bien que faciles à mettre en cuvre, rapides et peu coûteuses, posent des problèmes pour certains aliments (tourteaux, céréales...), le classement des aliments par la solubilité étant parfois différent de celui obtenu pour la dégradation in vivo ou in sacco. Les mesures utilisant du jus de rumen, " méthode in sacco » (Mehrez et Orskov, 1977), "fermentescibilité » in vitro (Verite et Demarquilly, 1978; RAAB et al., 1983) donnent probablement une image plus exacte de la dégradation dans le rumen, mais ne peuvent être appliquées dans les laboratoires de série, car elles nécessitent la présence d'animaux fistulisés du rumen. L'emploi de préparations enzymatiques pourrait pallier cet inconvénient, mais peu de méthodes sont proposées actuellement (Williams, 1986). Krishnamoorthy (1982), Genest (1982) utilisent une protéase bactérienne. Poos-Floyd, Klopfenstein et BritTON (1985) obtiennent une bonne prévision avec une protéase d'origine végétale. L'objectif de ce travail est de proposer une méthode de laboratoire simple, précise et applicable à tous les aliments et bien corrélée avec la méthode in sacco qui est actuellement utilisée comme base de calcul du système PDI en France (VERITE et al., 1987).

\section{Matériel et méthodes}

\section{A. Choix des préparations enzymatiques et tests effectués}

De nombreuses préparations enzymatiques sont disponibles sur le marché. Seules celles ayant déjà permis des résultats satisfaisants dans la bibliographie ont été testées. Trois enzymes commerciales protéolytiques ont été utilisées : 2 protéases bactériennes (Streptomyces griseus, type XIV, Sigma, pronase E. Merck), une protéase végétale (extraite de la ficine).

Pour les comparer, nous avons testé leur activité protéolytique sur du caséinate de sodium (Sigma C 8654) selon le protocole suivant : à $5 \mathrm{ml}$ d'une suspension de caséinate de sodium à $2 \mathrm{p}$. 100 dans du tampon borate phosphate $\mathrm{pH} 8(12,2 \mathrm{~g}$ de $\mathrm{Na}_{2} \mathrm{PO}_{4} \mathrm{H}_{2} 0 ; 8,91 \mathrm{~g}$ de $\mathrm{Na}_{2} \mathrm{~B}_{4} \mathrm{O}_{7} 10 \mathrm{H}_{2} \mathrm{O} /$ litre), maintenu dans un bain-marie à $40^{\circ} \mathrm{C}$, on ajoute $0,5 \mathrm{ml}$ de solution de protéase par tube. La concentration en protéase est de $0,1 \mathrm{mg} / \mathrm{ml}$ pour l'enzyme Sigma et Merck et $1 \mathrm{mg} / \mathrm{ml}$ pour la ficine. La réaction est arrêtée au bout de $10 \mathrm{mn}$ en ajoutant $5 \mathrm{ml}$ d'acide trichloracétique à $2 \mathrm{p} .100$ afin de précipiter les protéines. Au bout de $30 \mathrm{mn}$ (à $4^{\circ} \mathrm{C}$ le contenu des tubes est filtré sur filtre plissé et la densité optique du filtrat est mesurée à $280 \mathrm{~nm}$; les résultats sont exprimés en équivalent tyrosine. Le nombre d'unité/mg ou activité est le nombre d'équivalents tyrosine ( $\mu$ moles) libérés par minute dans les conditions du test. L'activité était respectivement de 2,66 UI/mg pour l'enzyme Sigma, de 3,07 UI/mg pour l'enzyme Merck et de 0,109 UI/mg pour la ficine. 
De plus, pour l'enzyme Sigma, nous avons testé :

1. l'influence de la concentration en enzyme : rapport enzyme/substrat,

2. l'influence de la solution tampon ( $\mathrm{pH}$, nature),

3. l'influence d'un pré-traitement cellulase-amyloglucosidase.

Dans la partie mise au point de la méthode, les résultats de dégradation enzymatique au temps $24 \mathrm{~h}$ sont comparés à la proportion d'azote dégradé in sacco, au temps $24 \mathrm{~h}$.

\section{B. Caractéristiques des matières premières}

12 matières premières différentes ont été retenues pour les tests de dégradation. Les matières premières choisies étaient représentatives des familles végétales et des principaux «types " d'ingrédients utilisés en alimentation animale : des céréales (orge,

\section{TABLEAU 1}

Caractéristiques des matières premières utilisées pour la mise au point de la méthode de dégradation enzymatique

Characteristics of feed ingredients used for enzymatic degradation tests

\begin{tabular}{|c|c|c|c|c|}
\hline \multirow{2}{*}{$\begin{array}{l}\text { Aliments } \\
\text { Feeds }\end{array}$} & \multicolumn{3}{|c|}{$\%$ MS } & \multirow{2}{*}{$\begin{array}{l}\text { Dégradabilité } \\
\text { théorique } \\
\text { (DT) (\% NT) } \\
\text { Theoretical } \\
\text { degradability } \\
\text { (DT) (\% NT) }\end{array}$} \\
\hline & $\stackrel{\mathrm{N}}{\text { Nitrogen }}$ & $\begin{array}{c}\text { CB } \\
\text { Crude fiber }\end{array}$ & $\begin{array}{l}\mathrm{ADF} \\
A D F\end{array}$ & \\
\hline $\begin{array}{l}\text { Luzerne déshydratée } \ldots \ldots \ldots \ldots \ldots \ldots \\
\text { Dehydrated luce, } .{ }_{2}\end{array}$ & 25,9 & 268 & 357,3 & 64,6 \\
\hline$\underset{\text { Barley }}{\text { Orge }} \ldots \ldots \ldots \ldots \ldots \ldots \ldots$ & 16,8 & 44 & 71,4 & 75,8 \\
\hline $\begin{array}{l}\text { Maïs . } \\
\text { Maize }\end{array}$ & 18,9 & 18 & 37,0 & 36,6 \\
\hline$\underset{\text { Oat }}{\text { Avoine }} \ldots \ldots \ldots \ldots \ldots \ldots \ldots$ & 18,8 & 141 & 201,1 & 82,7 \\
\hline $\begin{array}{l}\text { Son de blé } \ldots \ldots \ldots \ldots \ldots \ldots \ldots \ldots \\
\text { Wheat bran }\end{array}$ & 79,6 & 112 & 156,5 & 76,0 \\
\hline $\begin{array}{l}\text { Tourteau de soja } \ldots \ldots \ldots \ldots \ldots \ldots \ldots \\
\text { Soyabean meal }\end{array}$ & 97,3 & 64 & 85,0 & 64,4 \\
\hline $\begin{array}{l}\text { Tourteau de colza tanné } \\
\text { Treated rapeseed meal }\end{array}$ & 62,6 & 120 & 208,0 & 31,5 \\
\hline $\begin{array}{l}\text { Tourteau de colza } \\
\text { Rapeseed meal }\end{array}$ & 61,8 & 103 & 219,3 & 80,9 \\
\hline $\begin{array}{l}\text { Tourteau de tournesol } \\
\text { Sunflower meal }\end{array}$ & 65,9 & 162 & 221,7 & 80,3 \\
\hline $\begin{array}{l}\text { Graine de soja } \ldots \ldots \ldots \ldots \ldots \ldots \ldots \\
\text { Soya bean }\end{array}$ & 61,2 & 60 & 78,0 & 86,7 \\
\hline$\underset{\text { Lupin }}{\operatorname{Lupin}} \ldots \ldots \ldots \ldots \ldots \ldots \ldots \ldots$ & 57,6 & 126 & 177,0 & 93,9 \\
\hline $\begin{array}{l}\text { Féverole } \\
\text { Fieldbean }\end{array} \ldots \ldots \ldots \ldots \ldots \ldots \ldots$ & 42,5 & 93 & 131,0 & 85,3 \\
\hline
\end{tabular}


maïs, avoine) et leur sous-produit (son de blé), des tourteaux (soja, tournesol, colza, colza tanné), des graines de légumineuses (soja, lupin, féverole) et de la luzerne déshydratée. Leur composition chimique, ainsi que leur valeur de dégradabilité théorique (DT) in sacco étaient très variables (tabl. 1).

\section{Dégradabilité in sacco}

La dégradabilité théorique (DT) de l'azote a été calculée à partir de mesures de dégradation in sacco effectuées sur des vachés après des temps d'incubation de $2 \mathrm{~h}, 4 \mathrm{~h}$, $8 \mathrm{~h}, 16 \mathrm{~h}, 24 \mathrm{~h}, 48 \mathrm{~h}$ en tenant compte d'un taux de renouvellement des particules dans le réticulo-rumen de $0,06 \mathrm{~h}^{-1}$ (Michalet-Doreau, Verite et Chapoutor, 1987).

\section{Modélisation des résultats}

La méthode enzymatique a été appliquée en cinétique aux temps $(0 \mathrm{mn}, 5 \mathrm{mn}, 1 \mathrm{~h}$, $3 \mathrm{~h}, 5 \mathrm{~h}, 7 \mathrm{~h}, 24 \mathrm{~h}, 48 \mathrm{~h}$ ) sur les 12 aliments décrits. Afin de comparer la dégradabilité enzymatique de l'azote des aliments avec la dégradabilité théorique (DT) in sacco nous avons utilisé le modèle mathématique qui est celui d'Orskov et de McDonald (1979) sur l'ensemble de la période étudiée, soit $48 \mathrm{~h}$.

Une fraction (a) de l'aliment est immédiatement soluble, tandis qu'une fraction (b) est potentiellement dégradable selon une cinétique du $1^{\text {er }}$ ordre avec un taux horaire de dégradation (c). La quantité d'azote résiduelle est donc : $Y=(100-a-b)+b e^{(-c i)}$.

Comme pour la DT, pour calculer la dégradabilité enzymatique (DE) à partir des cinétiques d'hydrolyse des matières azotées par une enzyme, il faut également tenir compte du taux de sortie des particules du réticulo-rumen $k$ fixé comme pour la méthode in sacco à $0.06^{\mathrm{h}-1}$. On a donc $\mathrm{DE}=\frac{\mathrm{a}+\mathrm{bc}}{\mathrm{c}+\mathrm{k}}$

L'utilisation de 8 points de cinétique étant une mesure trop onéreuse pour les laboratoires de série, dans le but d'une application pratique pour ces laboratoires, les points de cinétique doivent être réduits et ceux retenus l'ont été après une analyse en régression descendante.

\section{Technique de solubilité}

La méthode de solubilité utilisée est la solubilité dans un tampon phosphate pH 6,9 (solubilité Durand, citée par Verite et Demarquilly, 1978). La dégradation enzymatique a été comparée à cette méthode. 


\section{Résultats}

\section{A. Mise au point de la méthode}

\section{Concentration en enzyme}

a) avec une quantité fixe de substrat (500 mg de M.S.)

4 concentrations en protéase (Sigma type XIV) ont été testées sur les 12 matières premières décrites dans le tableau $1: 0-0,5-1$ et $2 \mathrm{mg}$ enzyme $/ 500 \mathrm{mg}$ d'échantillon. Les résultats obtenus présentés dans la fig. 1 mettent en évidence l'influence très nette

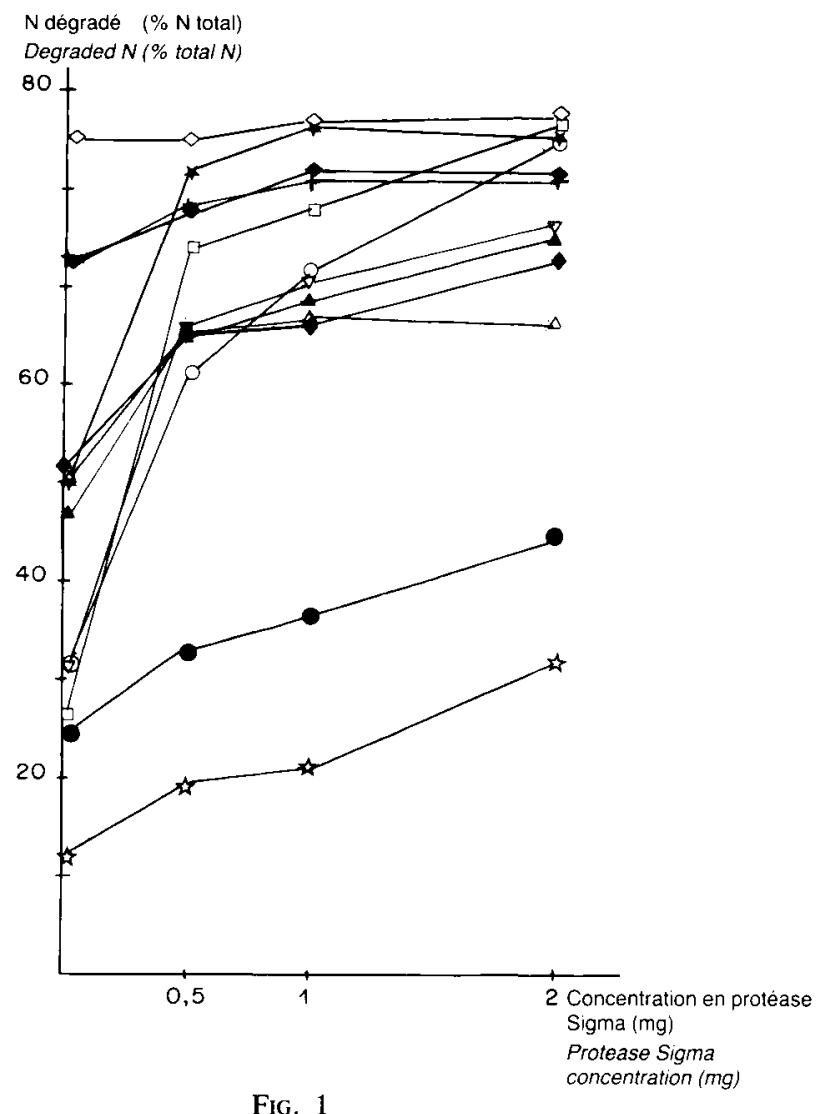

Azote dégradé à 24 heures ( $p .100 \mathrm{~N}$ total) en fonction de la concentration en protéase Sigma. Effects of protease Sigma concentration on nitrogen degradation at 24 hours ( $p .100$ total $N$ ).

$\diamond$ Lupin / Lupin.

$\square$ Tourteau de soja / Soyabean meal.

- Tourteau de tournesol / Sunflower meal.

Orge / Barley.

- Graine de soja crue / Soyabean:

+ Féverole / Fieldbean. $\nabla$ Luzerne déshydratée / Dehydrated lucerne

A Tourtcau de colza / Rapeseed meal.

- Son de blé/Wheat bran.

$\triangle$ Avoine / Oat.

- Maïs / Maize.

is Tourteau de colza tanné/Treated rapeseed meal. 
de l'accroissement de la concentration en enzyme sur le niveau de dégradation de l'azote des aliments, mais les réponses diffèrent nettement d'une matière première à l'autre ; si le " plateau " de la réponse est approximativement atteint pour la concentration de $0,5 \mathrm{mg} / 50 \mathrm{ml}$ de tampon, pour l'avoine et les graines oléo-protéagineuses (soja, lupin, féverole) il n'en est pas de même pour d'autres matières premières : ainsi pour les tourteaux de soja, colza, colza tanné, le «plateau " n'est pas encore atteint à la concentration de $2 \mathrm{mg} / 50 \mathrm{ml}$. En conséquence, le classement des aliments varie selon la concentration en enzyme.

Cependant, les pourcentages d'azote dégradé à 24 heures sont mieux corrélés avec ceux dégradés in sacco au temps $24 \mathrm{~h}$ pour une concentration en enzyme de $1 \mathrm{mg} / 50 \mathrm{ml}$ de tampon $(r=0,97 ; \mathrm{Sr}=4,1$ contre $\mathrm{r}=0,93 ; \mathrm{Sr}=6,4$ pour $2 \mathrm{mg} / 50 \mathrm{ml})$.

Dans la suite des essais, nous avons donc retenu la concentration en protéase Sigma type XIV de $1 \mathrm{mg} / 50 \mathrm{ml}$, soit 2,66 U. A cette concentration, le classement des aliments est le même que celui de l'in sacco à $24 \mathrm{~h}$, sauf pour la luzerne déshydratée et le tourteau de colza.

b) avec un rapport $\frac{\text { enzyme }}{\text { substrat }}$ constant (substrat isoazoté)

L'enzyme à la concentration retenue $(1 \mathrm{mg} / 50 \mathrm{ml})$ a été testée sur les 12 matières premières avec un rapport enzyme/substrat constant c'est-à-dire avec une concentration en substrat fixe de $20 \mathrm{mg} \mathrm{N}$ (Poos-Floyd, Klopfenstein et Britton, 1985).

Les pourcentages d'azote dégradé ont eu tendance à diminuer de façon variable pour les céréales (23,3 points pour l'orge ; 3,4 points pour le maïs ; 7,1 points pour l'avoine) et n'ont pratiquement pas varié pour les graines oléo-protéagineuses et les tourteaux. La liaison entre l'azote dégradé in sacco $24 \mathrm{~h}$ et la dégradation enzymatique au temps $24 \mathrm{~h}$ est fortement diminuée $\left(\mathrm{Sr}_{r}=8,0\right.$ contre 4,0) (tableau 2), et nous avons donc, par la suite, travaillé avec une teneur en substrat fixe de $500 \mathrm{mg}$ de M.S.

TABleaU 2

Liaison entre la dégradation enzymatique et la dégradation in sacco au temps 24 h pour 12 aliments Relationship between enzymatic degradation and in sacco degradation at $24 \mathrm{~h}$ for 12 feeds

\begin{tabular}{|c|c|c|c|c|}
\hline & $\begin{array}{c}\text { Tampon } \mathrm{BP} * \\
\text { pH } 8 \\
\text { Buffer } B P^{*} \\
\text { pH } 8\end{array}$ & $\begin{array}{l}\text { Tampon } \mathrm{P}^{* *} \\
\mathrm{pH} 8 \\
\text { Buffer } P^{* *} \\
\text { pH } 8\end{array}$ & $\begin{array}{c}\text { Tampon BP } \\
\text { pH } 6,3 \\
\text { Buffer } B P \\
\text { pH } 6.3\end{array}$ & $\begin{array}{c}\text { Tampon BP } \\
\text { pH } 8 \text { isoazoté } \\
\text { Buffer } B P \\
\text { pH } 8\end{array}$ \\
\hline $\begin{array}{l}\text { Dégradation enzymatique moyenne } \\
(\% \mathrm{Nt}) \ldots \ldots \ldots \ldots \ldots \ldots \\
\begin{array}{l}\text { Mean value for enzymatic } \\
\text { degradation }\end{array}\end{array}$ & 67,8 & 63,7 & 58,3 & 64,8 \\
\hline $\mathbf{r} \ldots \ldots \ldots \ldots \ldots \ldots \ldots \ldots$ & 0,97 & 0,92 & 0,89 & 0,89 \\
\hline$\underset{R S D}{\operatorname{Sr} \ldots \cdots \cdots \cdots \cdots \cdots \cdots}$ & 4,1 & 6,8 & 8,9 & 8,0 \\
\hline
\end{tabular}

* Borate-phosphate $\left(\mathrm{Na}_{2} \mathrm{~B}_{4} \mathrm{O}_{7} 10 \mathrm{H}_{2} \mathrm{O} 8,91 \mathrm{~g} / \mathrm{l}-\mathrm{NaH}_{2} \mathrm{PO}_{4} 2 \mathrm{H}_{2} \mathrm{O} 12.2 \mathrm{~g} / \mathrm{l}\right)$

** Phosphate $\left(\mathrm{Na}_{2} \mathrm{PO}_{4} 12 \mathrm{H}_{2} \mathrm{O} 71,64 \mathrm{~g} / 1-\mathrm{NaH}_{2} \mathrm{PO}_{4} 2 \mathrm{H}_{2} \mathrm{O} 31,21 \mathrm{~g} / 1\right)$ 


\section{Influence $d u p H$}

Nous avons testé l'influence du $\mathrm{pH}$ du tampon borate-phosphate : $\mathrm{pH} 8(\mathrm{pH}$ optimum d'activité de l'enzyme) et $\mathrm{pH}$ 6,3 qui correspond au $\mathrm{pH}$ moyen du rumen dans le cas d'une alimentation comprenant $70 \%$ de foin et $30 \%$ d'aliment concentré choisie pour les mesures in sacco. On observe, de façon générale, une diminution du pourcentage d'azote dégradé à $\mathrm{pH} 6,3$ pour les 12 aliments considérés. Cette diminution est en moyenne de 9,5 points, mais elle est très variable selon les matières premières considérées puisqu'elle peut varier de 1,6 pour le lupin à -11 points pour le tourteau de soja. Par ailleurs, la liaison avec les valeurs in sacco est diminuée $(\mathrm{Sr}=8,9$ à $\mathrm{pH} 6,3$ contre 4,1 à $\mathrm{pH} 8$ ).

\section{Influence de la solution tampon}

Deux solutions tampons ont été testées: un tampon borate-phosphate $\mathrm{pH} 8$ (Krishnamoorthy et al., 1983) et un tampon phosphate pH 8 (Genest, 1982) sur les 12 matières premières avec l'enzyme Sigma type XIV. La liaison avec les valeurs in sacco est meilleure avec le tampon borate-phosphate $\mathrm{pH} 8(\mathrm{Sr}=4,1)$ contre 6,8 avec le tampon phosphate $\mathrm{pH} 8$.

\section{Influence d'un pré-traitement}

Nous avons testé l'influence d'un pré-traitement cellulase-amyloglucosidase sur des matières premières riches en amidon ou en fibres. Ces enzymes (cellulase, amyloglucosidase) ayant des $\mathrm{pH}$ optimum d'activité à 4,6 , donc très différents de celui de la protéase, deux hydrolyses séparées ont été effectuées. Les matières premières choisies pour ce test étaient riches en constituants pariétaux (drèches de brasserie, pulpe de betterave, foin de luzerne), riches en amidon (maïs et orge) ou avec une teneur élevée en azote (tourteaux). Les pourcentages d'azote dégradé au temps $24 \mathrm{~h}$ figurent dans le tableau 3. Pour les aliments à teneur en constituants pariétaux élevée (drèches de brasserie) le pré-traitement augmente peu le pourcentage d'azote dégradé ; par contre, il est fortement augmenté dans le cas du tourteau de colza tanné, du maïs, du tourteau de soja et les pourcentages d'azote dégradé de ces deux derniers aliments semblent trop élevés par comparaison aux valeurs obtenues in sacco.

\section{Comparaison entre plusieurs types de préparations enzymatiques}

Nous avons comparé deux préparations enzymatiques d'origine bactérienne extraites de Streptomyces griseus (protéase type XIV Sigma et une protéase Merck) ainsi qu'une préparation enzymatique d'origine végétale (la ficine). Ces enzymes ont été testées à la même activité de $2,66 \mathrm{U}$ (tableau 4) sur les 12 matières premières utilisées précédemment. Les résultats de la fig. 2 indiquent que la dégradation au temps $24 \mathrm{~h}$ est pratiquement identique avec l'une ou l'autre des protéases d'origine bactérienne. Avec la « ficine », le classement des aliments est inchangé ; toutefois, la dégradation est en moyenne diminuée de 5 points, avec des écarts allant de $-17,2$ pour le tourteau de tournesol à $+12,7$ points pour le maïs.

La liaison entre le pourcentage d'N dégradé in sacco au temps $24 \mathrm{~h}$ et le pourcentage d'azote dégradé par la méthode enzymatique au temps $24 \mathrm{~h}$ est plus étroite avec la 


\section{TABLEAU 3}

Influence d'un pré-traitement cellulase + amyloglucosidase sur le pourcentage d'azote dégradé au temps $24 \mathrm{~h}$.

Influence of pre-treatment cellulase + amyloglucosidase on nitrogen degradation at $24 \mathrm{~h}$.

\begin{tabular}{|c|c|c|c|}
\hline \multirow[t]{2}{*}{$\begin{array}{l}\text { Aliments } \\
\text { Feeds }\end{array}$} & \multirow[t]{2}{*}{$\begin{array}{l}\mathrm{N} \text { total } \\
(\% \mathrm{MS}) \\
\text { Total nitrogen } \\
(\% \text { DM })\end{array}$} & \multicolumn{2}{|c|}{$\begin{array}{c}\text { Pourcentage d'azote dégradé } \\
(\% \text { N total) } \\
\text { Percentage of degraded nitrogen } \\
(\% \text { Total } N)\end{array}$} \\
\hline & & (Protéase seule) & (Protéase + amylase) \\
\hline $\begin{array}{l}\text { Drèche de brasserie } \ldots \ldots \ldots \\
\text { Brewers' grain }\end{array}$ & 52,1 & 52,0 & 64,9 \\
\hline $\begin{array}{l}\text { Drèche de brasserie } \ldots \ldots \ldots \ldots \\
\text { Brewers' grain }\end{array}$ & 49,6 & 47,0 & 51,1 \\
\hline $\begin{array}{l}\text { Tourteau de soja } \ldots \ldots \ldots \ldots \\
\text { Soyabean meal }\end{array}$ & 82,0 & 77,5 & 87,5 \\
\hline $\begin{array}{l}\text { Pois } \ldots \ldots \ldots \ldots \ldots \ldots \\
P e a\end{array}$ & 41,0 & 80,9 & 84,1 \\
\hline $\begin{array}{l}\text { Maïs } \ldots \ldots \ldots \ldots \ldots \\
\text { Maize }\end{array}$ & 15,5 & 33,0 & 79,0 \\
\hline $\begin{array}{l}\text { Foin de luzerne } \ldots \ldots \ldots \ldots \\
\text { Lucerne hay }\end{array}$ & 28,1 & 72,1 & 79,5 \\
\hline $\begin{array}{l}\text { Luzerne déshydratée } \ldots \ldots \ldots \ldots \\
\text { Dehydrated lucerne }\end{array}$ & 23,2 & 70,5 & 76,8 \\
\hline $\begin{array}{l}\text { Pulpe de betteraves } \ldots \ldots \ldots \\
\text { Sugar beet pulp }\end{array}$ & 15,2 & 73,9 & 72,4 \\
\hline$\underset{\text { Lupin }}{\operatorname{Lupin}} \ldots \ldots \ldots \ldots \ldots \ldots$ & 57,2 & 76,2 & 815 \\
\hline $\begin{array}{l}\text { Orge } \\
\text { Barley }\end{array} \ldots \ldots \ldots \ldots \ldots \ldots$ & 16,4 & 77,8 & 8.0 \\
\hline $\begin{array}{l}\text { Tourteau de colza } \ldots \ldots \ldots \\
\text { Rapeseed meal }\end{array}$ & 61,8 & 71,9 & 76.7 \\
\hline $\begin{array}{l}\text { Tourteau de colza tanné } \ldots \ldots \\
\text { Treated rapeseed meal }\end{array}$ & 62,6 & 26,3 & $=3,4$ \\
\hline
\end{tabular}

TABLEAU 4

Activité enzymatique des enzymes et concentration en enzymes utilisée pour les essais de dégradation des protéines.

Enzymatic activity and concentration used for protein degradation assays.

\begin{tabular}{|c|c|c|}
\hline $\begin{array}{l}\text { Enzyme } \\
\text { Enzyme }\end{array}$ & $\begin{array}{l}\text { Activité } \\
\text { UI/mg } \\
\text { Activity }\end{array}$ & $\begin{array}{c}\text { Concentration } \\
(\mathrm{mg} / \mathrm{ml}) \\
\text { Concentration }\end{array}$ \\
\hline Bacterial Protease (Streptomyces griseus Sigma) & 2,66 & 0,02 \\
\hline Bacterial Protease E (Streptomyces griseus Merck) & 3,07 & 0,017 \\
\hline Latex) & 0,109 & 0,49 \\
\hline
\end{tabular}



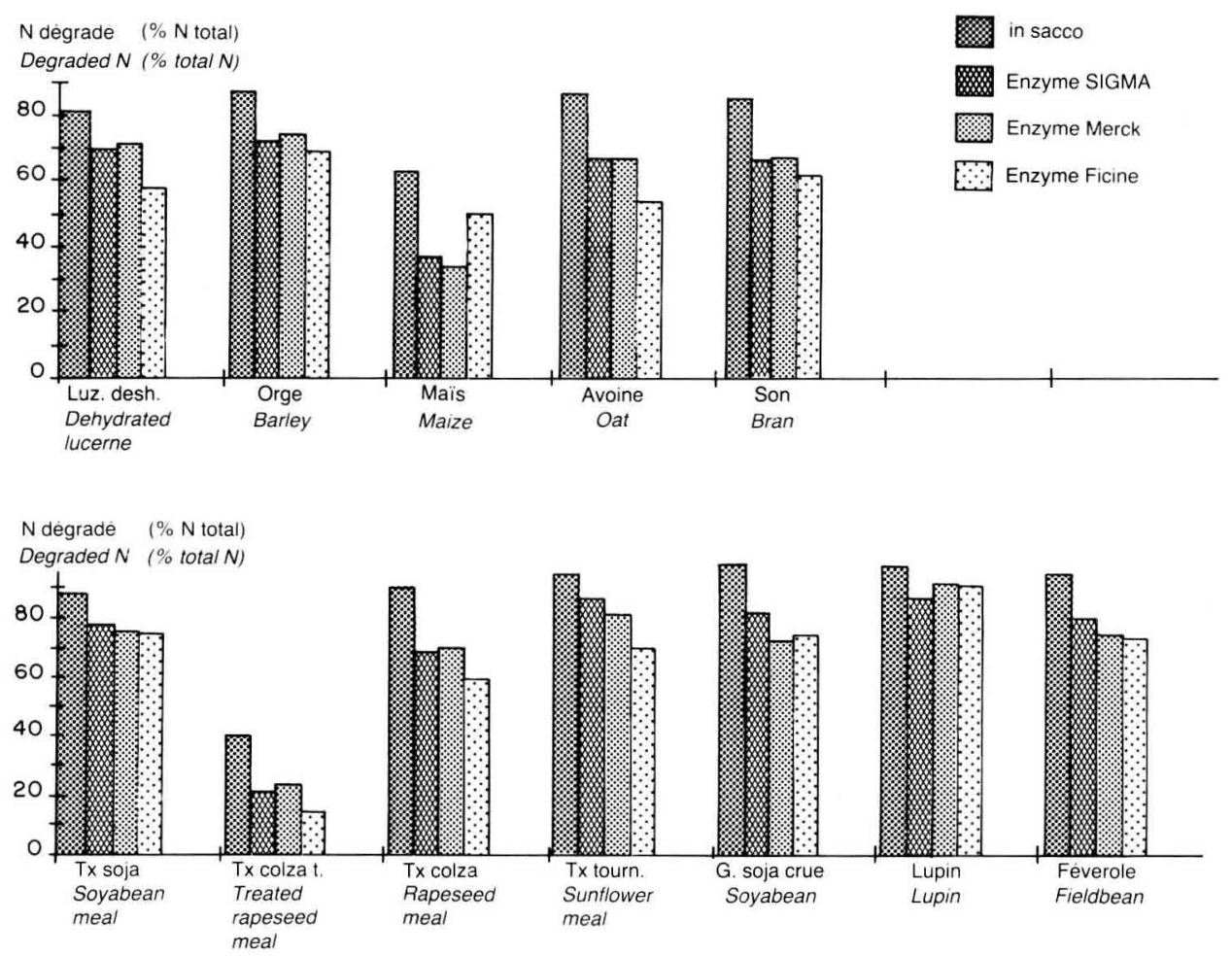

FIG. 2

Comparaison entre l'azote dégradé in sacco au temps 24 heures et l'azote dégradé par différentes enzymes au temps 24 heures.

Comparison between in sacco nitrogen degradability at 24 hours and nitrogen degradation by different enzymes at 24 hours.

protéase Sigma qu'avec les autres enzymes testées $(r=0,97 ; S r=4,1$ contre $r=0,94$; $\mathrm{Sr}=6,1$ avec la protéase « Merck» et $\mathrm{r}=0,92 ; \mathrm{Sr}=7,0$ avec la ficine).

\section{Méthode retenue}

La méthode retenue pour réaliser des comparaisons ultérieures avec les mesures in sacco ou d'autres méthodes de prédiction de la valeur azotée est décrite dans le tableau 5 .

La répétabilité intra-série (r) et la reproductibilité inter-série (e) de la méthode proposée ont été calculées à partir des déterminations en double sur la luzerne déshydratée témoin : les valeurs sont en moyenne respectivement de 0,005 et 0,03 . 


\section{TABleau 5 \\ Description du protocole de la méthode retenue. \\ Working method.}

L'échantillon subit une hydrolyse par une protéase d'origine bactérienne dans un tampon borate-phosphate $\mathrm{pH} 8$ aux temps $1 \mathrm{~h}$ et $24 \mathrm{~h}$. Le dosage de l'azote dégradé est effectué sur le surnageant par la méthode Kjeldhal.

Samples were hydrolysed with a bacterial protease in a borate-phosphate buffer at $\mathrm{pH} 8$ at I $\mathrm{h}$ and $24 \mathrm{~h}$. The nitrogen on the supernatant was measured using Kjeldhal method.

1) Pesée de $0,5 \mathrm{~g}$ d'échantillon broyé à la grille de $0,8 \mathrm{~mm}$ dans un tube à centrifuger de $80 \mathrm{ml}$ (chaque point de cinétique est réalisé en double). Parallèlement la teneur en matière sèche est déterminée en double à $103{ }^{\circ} \mathrm{C}$ pendant $48 \mathrm{~h}$. Un témoin est introduit dans chaque série (temps $1 \mathrm{~h}$ et $24 \mathrm{~h}$ ) ainsi que deux tubes sans échantillon (ce sont les blancs - incubation $24 \mathrm{~h}$ ) qui permettent de connaître la teneur en azote de la préparation enzymatique.

1) $0.5 \mathrm{~g}$ samples were weighed in centrifugation tubes of $80 \mathrm{ml}$ (duplicate samples were taken at each kinetic point). Dry matter content was measured with 2 samples oven dried at $103^{\circ} \mathrm{C}$ until they had reached a content weight. A control sample was hydrolysed at 1 and $24 \mathrm{~h}$ with each series and two tubes without samples were also incubated at $24 \mathrm{~h}$. This procedure was carried out to determine the nitrogen content of the enzymatic evolution.

2) Adjonction de $50 \mathrm{ml}$ de solution enzymatique.

Bouchage des tubes. Incubation dans un bain-marie thermostaté à $40^{\circ}$.

Agitation manuelle aux temps $0 \mathrm{mn}, 1 \mathrm{~h}, 2 \mathrm{~h}, 3 \mathrm{~h}, 4 \mathrm{~h}, 5 \mathrm{~h}, 6 \mathrm{~h}, 7 \mathrm{~h}$, puis $24 \mathrm{~h}$.

- Solution enzymatique ( $2 \mathrm{~g}$ d'enzyme $/ 1$ tampon borate-phosphate).

- tampon borate-phosphate pH $8\left(12,2 \mathrm{~g} / 1 \mathrm{NaH}_{2} \mathrm{PO}_{4} 2 \mathrm{H}_{2} 0 ; 8,91 \mathrm{~g} / \mathrm{l} \mathrm{Na} \mathrm{B}_{4} \quad 10 \mathrm{H}_{2} \mathrm{O}\right)$.

- Enzyme : protéase extraite de Streptomyces griseus (type XIV, Sigma ${ }^{\circ}$ P-5147) (dissoudre l'enzyme dans un tampon borate-phosphate $\mathrm{pH} \mathrm{8,} \mathrm{ajuster} \mathrm{la} \mathrm{solution} \mathrm{et} \mathrm{filtrer} \mathrm{ensuite} \mathrm{la}$ solution enzymatique obtenue (filtre Whatman $\mathbf{n}^{\circ} 41$ ). La solution doit être préparée extemporanément.

- Tétracycline (antibiotique) Sigma $\mathrm{n}^{\circ} \mathrm{T}-3258 \quad(10 \mathrm{mg} / 100 \mathrm{ml}$ tampon borate-phosphate ph 8). Cette solution peut être conservée au maximum 1 mois à $+4^{\circ} \mathrm{C}$.

- Nystatine (antifongique) (Sigma $\left.\mathrm{n}^{\circ} 3503\right)(10 \mathrm{mg} / \mathrm{l})$. La nystatine est pesée directement.

Préparation enzymatique (pour $500 \mathrm{mg}$ d'échantillon).

- $0,5 \mathrm{ml}$ de solution enzymatique $(1 \mathrm{mg} / 500 \mathrm{mg}$ d'échantillon).

- $0,5 \mathrm{ml}$ de solution de tétracycline $(0,05 \mathrm{mg} / 500 \mathrm{mg}$ d'échantillon).

- $0,5 \mathrm{ml}$ nystatine $(0,5 \mathrm{mg} / 500 \mathrm{mg}$ échantillon).

Compléter à $50 \mathrm{ml}$ avec du tampon borate-phosphate $\mathrm{pH} 8$.

2) Addition of $50 \mathrm{ml}$ of enzymatic solution (1). The tubes were sealed and left to incubate in a thermostated waterbath at $40^{\circ} \mathrm{C}$. Enzymatic solution $(2 \mathrm{~g}$ enzyme/l borate-phosphate buffer).

- Borate-phosphate buffer pH $8\left(12,2 \mathrm{~g} / \mathrm{l} \mathrm{NaH}_{2} \mathrm{PO}_{4} 2 \mathrm{H}_{2} \mathrm{O} ; 8.91 \mathrm{~g} / \mathrm{l} \mathrm{Na}_{2} \mathrm{~B}_{4} 10 \mathrm{H} \mathrm{H}_{2} \mathrm{O}\right)$.

- Enzyme : protease extracted from Streptomyces griseus (type XIV, Sigma $n^{\circ} P-5147$ ) (Dissolve the enzyme in a borate-phosphate buffer, adjust the solution and filter it (filter Whatman $n^{o} 41$ ), This preparation was used immediately.

- Tetracycline (antibiotic) (Sigma $\mathrm{n}^{\circ} 3503$ ) (10 mg/100 ml borate-phosphate buffer pH 8). supply.

This preparation can be stored a month in a refrigerator at $4{ }^{\circ} \mathrm{C}$ was used with a month of

- Nystatine (antifungal) (Sigma $\left.n^{\circ} 3503\right)(10 \mathrm{mg} / \mathrm{l})$.

This preparation was used immediately.

Enzymatic preparation (500 mg of sample).

- $0.05 \mathrm{ml}$ of enzymatic solution $(1 \mathrm{mg} / 500 \mathrm{mg}$ of sample).

- $0.05 \mathrm{ml}$ of tetracycline solution $(0.05 \mathrm{mg} / 500 \mathrm{mg}$ of sample $)$.

- $0.5 \mathrm{mg}$ of nystatine $(0.5 \mathrm{mg} / 500 \mathrm{mg}$ of sample)

in $50 \mathrm{ml}$ of borate-phosphate buffer at pH 8 .

3) Après retrait des tubes aux temps $1 \mathrm{~h}$ et $24 \mathrm{~h}$, centrifuger $5 \mathrm{mn}$ à 3000 tours $/ \mathrm{min}$. $5 \mathrm{mn}$

3) Tubes removed at the times $1 \mathrm{~h}$ and $24 \mathrm{~h}$ were then centrifugated at 3000 turn/mn over 
4) Filtration sur filtres (Durieux 2B) préalablement humidifiés avec de l'eau distillée. water.

4) Filtration on filter papers (Durieux $2 B$ ) which were previously humidified with distilled

5) Dosage de l'azote sur le surnageant par la méthode Kjeldhal.

5) Nitrogen measure on the supernatant using Kjeldhal method.

6) Calcul

Les valeurs d'azote dégradé sont rapportées d'abord à la matière sèche de départ, puis en p. 100 de l'azote de l'échantillon.

Quantité d'azote dégradé dans le surnageant (en p. $100 \mathrm{MS})=\frac{14 \times \mathrm{T} \times(\mathrm{V}-\mathrm{Vo})}{\mathrm{m} \times 1000} \times 100$

$\mathrm{T}$ : normalité de lacide chlorhydrique.

$\mathrm{V}$ : volume en $\mathrm{ml}$ d'acide chlorhydrique nécessaire à la neutralisation pour la prise d'essai.

Vo : volume en $\mathrm{ml}$ d'acide chlorhydrique nécessaire à la neutralisation pour le blanc.

$\mathrm{m}$ : masse en $\mathrm{g}$ de la prise d'essai (exprimée par rapport à la MS).

Quantité d'azote dégradé dans le surnageant (en p. $100 \mathrm{~N}$ ) $)=$

quantité d'azote dégradé dans le surnageant (p. 100 MS)

teneur en $\mathrm{N}$ total (en p. $100 \mathrm{MS}$ )

Chaque série est corrigée par le témoin.

6) Calculations

The nitrogen degradability was firstly reported to the initial DM content of the sample and then it was then expressed as a p. 100 of the total $N$ content of the sample.

Amount of $N$ degraded in the supernatant $(\%$ dry matter $)=\frac{14 \times T \times(V-V o)}{m \times 1000} \times 100$

$\mathrm{T}$ : normality of $\mathrm{HCl}$.

$V$ : volume of $\mathrm{HCl}$ (in $\mathrm{ml}$ ) necessary to neutralize the sample.

Vo: volume of $\mathrm{HCl}$ (in $\mathrm{ml}$ ) necessary to neutralise the blank.

$m$ : weight of the sample ( $p .100$ dry matter).

Amount of $N$ degraded in the supernatant (in p. $100 \mathrm{Nt}$ ) $=$

amount of $N$ degraded in the supernatant (p. $100 \mathrm{DM}$ )

total $N$ content (in p. 100 of DM)

Each serie was adjusted with the control.

\section{B. Comparaison entre la D.T. \\ et la dégradation enzymatique ou la solubilité Durand}

\section{Etude en cinétique}

La méthode retenue a été appliquée aux temps $0 \mathrm{mn}, 5 \mathrm{mn}, 1 \mathrm{~h}, 3 \mathrm{~h}, 5 \mathrm{~h}, 7 \mathrm{~h}$, $24 \mathrm{~h}$ et $48 \mathrm{~h}$ aux 12 aliments décrits. Les courbes de dégradation entre 0 et $48 \mathrm{~h}$ (fig. 3) et les résultats du tableau 6 mettent en évidence des différences de dégradation entre les groupes de matières premières et parfois à l'intérieur d'un même groupe tant au niveau de la fraction dégradable (immédiatement soluble et dégradée par les enzymes) que du taux horaire de dégradation.

L'azote des céréales est dégradé différemment suivant la matière première considérée : le maïs est beaucoup moins dégradé que les autres céréales (avoine et orge). La fraction a (azote immédiatement soluble) est très importante pour les graines protéagineuses ou oléoprotéagineuses (féverole, lupin, soja).

Au temps $48 \mathrm{~h}$, en moyenne 75 p. 100 de l'azote des tourteaux est dégradé. La fraction immédiatement soluble (a) est très variable $(10 \mathrm{p}, 100$ de l'azote total pour le 


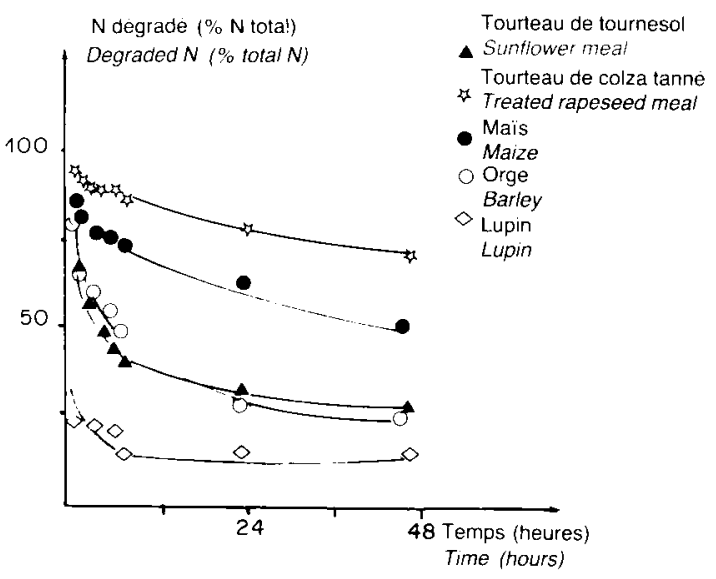

Fig. 3

Cinétique de dégradation de l'azote par la méthode enzymatique pour quelques aliments.

Kinetics of nitrogen degradation with enzymatic method for some feeds.

tourteau de soja et 35 à $40 \mathrm{p} .100$ pour les autres tourteaux) tandis que la fraction potentiellement dégradable disparaît à un taux horaire variant de $0,29 \mathrm{~h}^{-1}$ pour le tourteau de colza à $0,99 \mathrm{~h}^{1}$ pour le tourteau de tournesol. Le tourteau de colza tanné a une dégradation faible (d'environ $25 \mathrm{p} .100$ ), la fraction a est très diminuée par rapport à celle du tourteau de colza non traité $(a=6,2 p .100$ contre 34,9 p. 100).

\section{Liaison entre la D.T. et la dégradabilité enzymatique ou la solubilité (Durand) pour} les 12 aliments

Lorsque les 8 points de cinétique de la méthode enzymatique sont modélisés selon le modèle mathématique décrit par Orskov et MCDonald (1979), la dégradabilité enzymatique (DE) est corrélée à la DT par l'équation suivante :

$$
\begin{aligned}
& \mathrm{DT}=0,91 \mathrm{DE}+15,10 \\
& \mathrm{n}=12 \quad \mathrm{r}=0,93 \quad \mathrm{Sr}=7,39
\end{aligned}
$$

Pour les 12 aliments étudiés, la DE est inférieure à la DT de 15 points (la pente de l'équation n'est pas significativement différente de 1). Certains aliments s'écartent notablement de la droite de régression : l'avoine, le tourteau de colza ne sont pas assez dégradés par les enzymes, alors que le tourteau de soja l'est trop.

Dans le but d'une utilisation pratique de la méthode pour les laboratoires de série, par une analyse en régression descendante, on n'a pu retenir que deux points de cinétique, la dégradation enzymatique (Denz en p. 100 de l'azote total) aux temps $1 \mathrm{~h}$ et $24 \mathrm{~h}$ qui sont liées à la D.T. par l'équation suivante :

$$
\text { DT }=0,43 \text { Denz. T } 1 \mathrm{~h}+0,47 \text { Denz. T } 24 \mathrm{~h}+18,29 \quad \mathrm{r}=0,95 \quad \mathrm{Sr}=7,03
$$

La hiérarchie des 12 aliments est globalement la même que lorsque l'on prend en compte tous les points de la cinétique.

Avec la solubilité Durand, l'écart-type de la régression est beaucoup plus élevé $(\mathrm{Sr}=12,25 ; \mathrm{r}=0,80)$. 


\section{TABleaU 6}

Paramètres des cinétiques de dégradation enzymatique, dégrabilité enzymatique (DE) et dégradabilité théorique (DT).

Parameters of enzymatic degradability kinetics, enzymatic degradability (DE) and theoretical degradability (DT).

\begin{tabular}{|c|c|c|c|c|c|}
\hline $\begin{array}{l}\text { Aliments } \\
\text { Feeds }\end{array}$ & $(\% \stackrel{a}{N t})$ & $(\% \mathrm{~b}$ & $\underset{\left(h^{-1}\right)}{c}$ & $\mathrm{DE}=0,06 \mathrm{~h}^{-1}$ & $\begin{array}{l}\text { DT in sacco } \\
k=0,06 \mathrm{~h}^{-1}\end{array}$ \\
\hline $\begin{array}{l}\text { Luzerne déshydratée } \\
\text { Dehydrated lucerne }\end{array}$ & 28,2 & 43,1 & 0,15 & 59,1 & 64,6 \\
\hline$\underset{\text { Barley }}{\text { Orge }} \ldots \ldots \ldots \ldots \ldots$ & 24,9 & 53,5 & 0,11 & 59,6 & 75,8 \\
\hline$\underset{\text { Maize }}{\text { Mais }} \ldots \ldots \ldots \ldots \ldots$ & 16,5 & 32,0 & 0,06 & 32,5 & 36,6 \\
\hline $\begin{array}{l}\text { Avoine } \\
\text { Oat }\end{array}$ & 39,4 & 25,6 & 0,48 & 62,2 & 82,7 \\
\hline$\underset{\text { Bran }}{\text { Son }} \ldots \ldots \ldots \ldots \ldots \ldots$ & 40,9 & 25,4 & 1,23 & 65,1 & 76,0 \\
\hline $\begin{array}{l}\text { Tourteau de soja } \ldots \ldots \ldots \\
\text { Soyabean meal }\end{array}$ & 9,6 & 69,9 & 0,27 & 66,7 & 64,4 \\
\hline $\begin{array}{l}\text { Tourteau colza tanné } \quad \cdots \cdots \\
\text { Treated rapeseed meal }\end{array}$ & 6,2 & 31,3 & 0,03 & 16,7 & 31,5 \\
\hline $\begin{array}{l}\text { Tourteau colza } \ldots \ldots \ldots \ldots \\
\text { Rapeseed meal }\end{array}$ & 34,9 & 34,4 & 0,24 & 62,4 & 80,9 \\
\hline $\begin{array}{l}\text { Tourteau de tournesol } \ldots . . \\
\text { Sunflower meal }\end{array}$ & 38,7 & 45,1 & 0,79 & 80,7 & 80,3 \\
\hline $\begin{array}{l}\text { Graine de soja cru } \ldots \ldots \ldots \\
\text { Soyabean }\end{array}$ & 51,9 & 28,2 & 1,68 & 79,2 & 86,7 \\
\hline $\begin{array}{l}\text { Lupin } \\
\text { Lupin }\end{array}$ & 76,9 & 9,3 & 0,15 & 83,6 & 93,9 \\
\hline $\begin{array}{l}\text { Féverole } \ldots \ldots \\
\text { Fielbean }\end{array}$ & 72,9 & 9,1 & 0,30 & 80,5 & 85,3 \\
\hline
\end{tabular}

\section{Discussion}

La précision de la prévision de la dégradabilité de l'azote est meilleure avec la protéase Sigma, extraite de Streptomyces griseus, qu'avec les 2 autres enzymes testées. Cette enzyme, bien que différente des protéases du rumen (MaHAdevan et al., 1987 ; Laycock, Hazlewood et Miller, 1985) a été retenue dans les essais de méthodes in vitro par de nombreux auteurs (Pichard et VAN Soest, 1977 ; Krishnamoorthy, 1982 ; Nocek, Herbein et Polan, 1983 ; Chen et al., 1987). En revanche Poos-Floyd, KLOPFENSTEIN et BRITTON (1985) ont trouvé une meilleure prévision avec la ficine (extraite du latex), ce qui est en contradiction avec leurs conclusions de 1980, mais leur dernière étude ne porte que sur quatre aliments. Comme l'ont souligné SidDons et al. (1985), la proportion d'azote dégradée par les enzymes protéolytiques pour une durée 
d'incubation donnée est très dépendante de la nature de l'enzyme et de la nature du substrat. Il est donc nécessaire, pour prévoir au laboratoire la dégradabilité de l'azote des aliments, d'utiliser des enzymes peu spécifiques avec des spectres d'activité le plus large possible. La concentration en enzyme retenue de $1 \mathrm{mg} / 500 \mathrm{mg}$ d'échantillon, soit 2,6 U est peu différente de celle utilisée par Krishnamoorthy et al. (1983) (3,3 U). A cette faible concentration, une étude en cinétique est possible et permet une meilleure prévision de la dégradabilité de l'aliment. Cette étude en cinétique a d'ailleurs été utilisée par d'autres auteurs (Krishnamoorthy 1982 ; Poos Floyd, Klopfenstein et BRITTON, 1985 ; Siddons et al., 1985).

La dégradabilité de l'azote d'un aliment dépend des formes de protéines de réserve de l'aliment (Wallace, Broderick et Brammall, 1987) et de la localisation des protéines à l'intérieur des parois cellulaires (TAmmingA, 1983). Pour des matières premières riches en constituants pariétaux (drèches de brasserie, pulpe, foin de luzerne) ou riches en amidon (maïs) un pré-traitement cellulase plus amyloglucosidase n'a cependant pas apporté l'amélioration souhaitée puisque la dégradabilité a peu varié sauf pour le maïs où on observe une dégradation trop élevée par rapport aux valeurs in sacco habituellement trouvées (OrSkov, Fraser et McDonald, 1972).

En prenant en compte l'ensemble de la cinétique ( 8 points) ou seulement 2 points, la liaison ne varie pas, ce qui montre que l'on peut prévoir la DT par un point (temps $1 \mathrm{~h})$ qui représente pratiquement l'azote immédiatement soluble et un point plus éloigné (temps $24 \mathrm{~h}$ ) qui représente l'azote non soluble mais dégradable par les enzymes.

Si on ne prend en compte que 2 points de cinétique, la liaison entre la dégradabilité in sacco et la dégradation enzymatique est de $\mathrm{r}=0,95 ; \mathrm{Sr}=7,03$ pour 12 aliments. Cette liaison pas très étroite $(\mathrm{CV}=9,8 \mathrm{p}$. cent) résulte peut-être en partie du fait que les enzymes utilisées ne sont pas des enzymes du rumen, quoique MADSEN et Hvelplund (1985) aient obtenu également une faible corrélation avec la D.T. en utilisant du jus de rumen. Mais elle résulte peut-être aussi des imperfections de la mesure in sacco elle-même : une fraction de l'azote disparaissant des sachets résulte de pertes de particules qui peuvent être importants pour certaines matières premières telle que l'avoine (B. Michalet-Doreau, communication personnelle). De plus l'azote non dégradé restant dans les sachets correspond non seulement à l'azote non dégradé de l'aliment, mais aussi à l'azote des microbes fixés sur les particules restant dans les sachets. On pourra, de toute façon, tenir compte du fait que certains aliments s'écartent notablement de la droite de régression, en proposant dans l'avenir pour ces aliments un $\Delta$ sur l'ordonnée à l'origine si ces écarts se répètent et se confirment quand nous disposerons de plus de résultats.

Quoiqu'il en soit, l'utilisation d'enzymes présente l'avantage d'éviter l'entretien d'animaux porteurs de fistules; la prise en compte de seulement 2 points de cinétique permet une simplification considérable de la méthode et son utilisation par les laboratoires de série. De plus, cette méthode permet une précision meilleure par rapport à la solubilité ( $\mathrm{Sr}=7,0$ contre 12,3). L'étude de la dégradation enzymatique par cette technique est actuellement en cours sur d'autres aliments (aliments simples, aliments composés). 


\section{Summary \\ Prediction of feeds nitrogen degradability in the rumen by means of an enzymatic procedure}

The extent to which dietary proteins are absorbed in the small intestine of ruminants is dependent upon the amount and the rumen degradability of crude protein in the feed. The degradability of feeds nitrogen is calculated from kinetics of in sacco degradation, taking into account the rate of particle exit from the rumen as $0.06 /$ hour. However, there are potentials for laboratory methods which are cheaper and more easily carried out.

We propose a method based on nitrogen hydrolysis over 1 and $24 \mathrm{~h}$ with a bacterial protease in a borate-phosphate buffer maintained at $\mathrm{pH}$ 8. The enzymatic concentration (of Sigma type XIV), which was finally retained, was of $1 \mathrm{mg} / 50 \mathrm{ml}$ of buffer added to a fixed quantity of substrate rather than to an iso-nitrogenous substrate.

The effects of $\mathrm{pH}$, the type of buffer used and of a pre-treatment were investigated. The prediction of theoretical degradability (DT) was more accurate when the Sigma type XIV enzyme was used as compared to the other enzymes which were tested : "protease Merck » and «Ficine » (table 2).

This laboratory method (table 5), applied on 12 different feeds, enabled the prediction of DT to be made with more accuracy than when the usual method was used (i.e. solubility in a phosphate buffer at $\mathrm{pH} 6.9$ ).

Key words: Feeds, ruminant, theoretical degradability, nitrogen, enzymatic method.

\section{Références bibliographiques}

Chen G., SNiffen C.J., Russell J.B., 1987. Concentration and estimated flow of peptides from the rumen of dairy cattle : effects of protein quantity, protein solubility, and feeding frequency. J. Dairy Sci., 70, 983-992.

Crooker B.A., Sniffen C.J., Hoover W.H., Johnson L.L., 1978. Solvents for soluble nitrogen measurements in feedstuffs. J. Dairy Sci., 61, 437-447.

Genest C., 1982. Mise au point d'une méthode d'estimation in vitro de la dégradation des protéines dans le rumen et sa relation avec la détermination in vivo du flux d'azote alimentaire à l'entrée de l'intestin grêle. Thèse présentée à l'Université de Clermont II (U.E.R. de Recherche). 113 pages.

Henderickx H., Martin J., 1963. In vivo study of the nitrogen metabolism in the rumen. Compies-rendus de Recherches. Sci. Ind. Agr. Bruxelles, 31, I.

Krishnamoorthy U., 1982. Development of an in vitro technique to estimate rumen escape nitrogen in feedstuffs. Thesis presented at the Faculty of the Graduate School of Cornell University. 169 pages.

Krishnamoorthy U., Muscato T.V., Sniffen C.J., Van Soest P.J., 1982. Nitrogen fractions in selected feedstuffs. J. Dairy Sci. , 65, 217-225.

Krishnamoorthy U., Sniffen C.J., Stern M.D., Van Soest P.J., 1983. Evaluation of a rumen dynamic mathematical model and in vitro simulated rumen proteolysis to estimate the undegraded dietary nitrogen content of the feedstuffs. Br. J. Nutr., 50, 555-568.

Laycock K.A., Hazlewood G.P., Miller E.L., 1985. Potential use of proteolytic rumen bacteria for assessing feed protein degradability in vitro. Proc. Nutr. Soc., 44 (1), 54 A.

Madsen J., Hvelplund T., 1985. Protein degradation in the rumen. Acta Agric. Scand. Suppl., 25, 103-124.

Mahadevans S., Sauer F.D., Erfle J.D., 1987. Preparation of protease from mixed rumen microorganisms and its use for the in vitro determination of the degradability of true protein in feedstuffs. Can. J. Anim. Sci., 67, 55-46. 
Mehrez A.Z., Orskov E.R., 1977. A study of the artificial fibre bag technique for determining the digestibility of feeds in the rumen. J. Agric. Sci. Camb., 88, 645-650.

Michalet-Doreau B., Verite R., Chapoutot P., 1987. Méthodologie de mesure de la dégradabilité in sacco de l'azote des aliments dans le rumen. Bull. Tech. C.R.Z.V. Theix, INRA, 69, 57.

Nocek J.E., Herbein J.H., Polan C.E., 1983. Total amino acid release rates of soluble and insoluble protein fractions of concentrate feedstuffs by Streptomyces griseus. J. Dairy Sci., 66, 1663-1667.

Orskov E.R., Fraser R.C., McDonald, 1971. Digestion of concentrates in sheep. Effects of rumen fermentation of barley and maize diets on protein digestion. Brit. J. Nutr., 26, 477486.

Orskov E.R., MCDonald I., 1979. The estimation of protein degradability in the rumen from incubation measurements weighted according to rate of passage. J. Agric. Sci. Camb., 12, 499-503.

Pichard G., Van Soest P.J., 1977. Protein solubility of ruminant feeds. Cornell Nutr. Conf., 9198.

Poos M., Klopfenstein T., Britton R.A., Olson, 1980. An enzymatic technique for determining ruminal protein degradation. J. Dairy Sci, 63, (suppl. 1) : 141 (Abstr.).

Poos-Floyd M., Klopfenstein T., Britton R.A., 1985. Evaluation of laboratory techniques for predicting ruminal protein degradation. J. Dairy Sci., 68, 829-839.

Raab L., Cafantaris B., Jilg T., Menke K.H., 1983. Rumen protein degradation and biosynthesis. 1 - A new method for determination of protein degradation in rumen fluid in vitro. $B r . J$. Nutr., 50, 569-582.

Siddons R.C., Paradine J., Gale D.L., Evans R.T,, 1985. Estimation of the degradability of dietary protein in the sheep rumen by in vivo and in vitro procedures. Brit. J. Nutr., 54, 545561.

TAmminga S., 1983. Recent advances in our knowledge on protein digestion and absorption in ruminants. IVth Int. Symp. Protein metabolism and nutrition, Clermont-Ferrand (France). 59 septembre 1983. p. 263-287. Ed. INRA Publ., 1983, Vol. I (Les Colloques de l'INRA, $n^{\circ}$ 16).

Verite R., Demarquilly C., 1978. Qualités de matières azotées des aliments pour ruininants. In "La vache laitière », 143-157. Ed. INRA Publ. Route de Saint-Cyr, 78000 Versailles.

Verite R., Michalet-Doreau B., Chapoutot P., Peyraud J.L., Poncet C., 1987. Révision du système des protéines digestibles dans l'intestin (P.D.I.). Bull. Techn. C.R.Z.V. Theix, INRA, 70, 19-34.

Waldo D.R., Goering H.K., 1979. Insolubility of proteins in ruminant feeds by four methods. J. Anim. Sci., 49, 1560-1568.

Wallace R.J., Broderick G.A., Brammal M.L., 1987. Protein degradation by ruminal microorganisms from sheep fed dietary supplements of urea, casein, or albumin. Aipl. Environ. Microbial., 53, 751-753.

Williams A.P., 1986. Laboratory methods of determining protein degradability in ruminants. Research and Development in Agriculture, 3, 1, 1-5.

Wohlt J.E., SNIFFen C.J., Hoover W.H., 1973. Measurements of protein solubility in common feedstuffs. J. Dairy Sci., 56, 1052-1057. 